\title{
Profil Socio-Économique Et Démographique Des Populations Issues De La Migration Sur Le Littoral Ôdjoukrou. (Sud De La Côte d'Ivoire)
}

\author{
Mel Mélèdje Melaine \\ Doctorant à l'Institut de Géographie Tropicale (IGT), Université Félix \\ Houphouët Boigny d'Abidjan, Côte d'Ivoire

\section{Aloko N'guessan Jérôme} \\ Directeur de Recherche à l'Institut de Géographie Tropicale (IGT) \\ Université Félix Houphouët Boigny d'Abidjan, Côte d'Ivoire
}

Doi: 10.19044/esj.2018.v14n5p359 URL:http://dx.doi.org/10.19044/esj.2018.v14n5p359

\begin{abstract}
The rural country of ôdjoukrou, or lôdjoukrou, is part of the Ivorian coastline. It is a space with enormous economic potential. Agricultural space, the boom in the plantation economy, will mobilize a large foreign workforce. This paper focuses on analyzing the socio-economic and demographic characteristics of populations resulting from migration. To meet the objective of this study, the methodological approach used is based on a literature search. We also used a field survey motivated by a questionnaire administered to 1000 migrants. An interview was also conducted with the chieftaincies of the various localities. The study reveals that the majority of migrants are West African immigrants with $79.8 \%$. The majority of migrants, estimated at $38 \%$, work in agriculture. In addition, the study allows us to relate the type of activity carried out in relation to the migrant's geographical origin. Most unhealthy incomes are considered satisfactory, to the point of encouraging regular monthly transfers to other family members of the starting spaces.
\end{abstract}

Keywords: Profile, socio-economic, demographic, migration, ôdjoukrou, Ivory Coast

\section{Résumé}

Le pays rural ôdjoukrou, ou encore lôdjoukrou, s'inscrit dans le littoral ivoirien. C'est un espace aux potentiels économiques énormes. Espace à vocation agricole, l'essor de l'économie de plantation va y mobiliser une abondante main d'œuvre étrangère. L'objectif de cette étude est d'analyser les caractéristiques socio-économiques et démographiques des populations issues de la migration. 
Pour répondre à cet objectif, l'approche méthodologique se base sur une recherche documentaire. Nous avons aussi eu recours à une enquête de terrain motivée par un questionnaire administré à 1000 migrants et un entretien avec les chefferies des différentes localités investiguées.

L'étude révèle que les migrants sont majoritairement des allogènes Ouest africains avec une proportion de $79,8 \%$. La majorité des migrants, estimée à $38 \%$ travaillent dans l'agriculture. En outre, l'étude nous permet de mettre en relation le type d'activité exercée en rapport avec l'origine géographique du migrant. Les revenus non thésaurisés sont en majorité jugés satisfaisants, jusqu'à favoriser des transferts mensuels réguliers vers d'autres membres de la famille des espaces de départ.

Mots clés : Profil, socio-économique, démographique, migration, ôdjoukrou, Côte d'Ivoire

\section{Introduction}

La Côte d'Ivoire est un pays d'Afrique occidentale qui fait partie des principaux pôles d'accueil de migrants en Afrique (OIM, 2009).

Son domaine littoral, siège d'une importante pratique agricole est un espace clé dans la vie économique du pays. Pour KABLAN (2005) il s'agit du plus grand marché potentiel de consommateurs et la zone qui concentre les $4 / 5^{\mathrm{ème}}$ des industries du pays, ainsi que la moitié de ses richesses. D'importants projets agro-industriels y ont été développés en l'occurrence dans le pays ôdjoukrou dont la capitale Dabou est à quelques encablures d'Abidjan. De par sa position géographique stratégique relativement à son statut d'espace carrefour et de l'importance de ses terres cultivables, le pays ôdjoukrou offre des potentialités au développement de l'économie de plantation.

Ainsi, un afflux de populations issues de l'hinterland et des contrées sahéliennes y est observé depuis la période coloniale. La crise économique des années 1980, et l'avènement des crises politico militaires des années 2000, va changer la configuration sociale, démographique et économique de lôdjoukrou. Des questions identitaires en rapport avec la pratique agricole et la possession foncière ont perturbé les équilibres sociaux.

Nous nous engageons dans le présent article à porter réflexion sur les conditions d'adaptation des migrants à ce nouveau contexte social de l'espace ôdjoukrou. Pour ce faire, une étude du profil de ces derniers nous parait nécessaire.

\section{Méthodologie}

Le champ d'expérimentation du présent article est le pays rural ôdjoukrou. Il peut être assimilé au département de Dabou, dans le sud de la Côte d'Ivoire comme nous le confère la figure 1. 
Figure 1 : Localisation du département de Dabou dans le polygone ivoirien

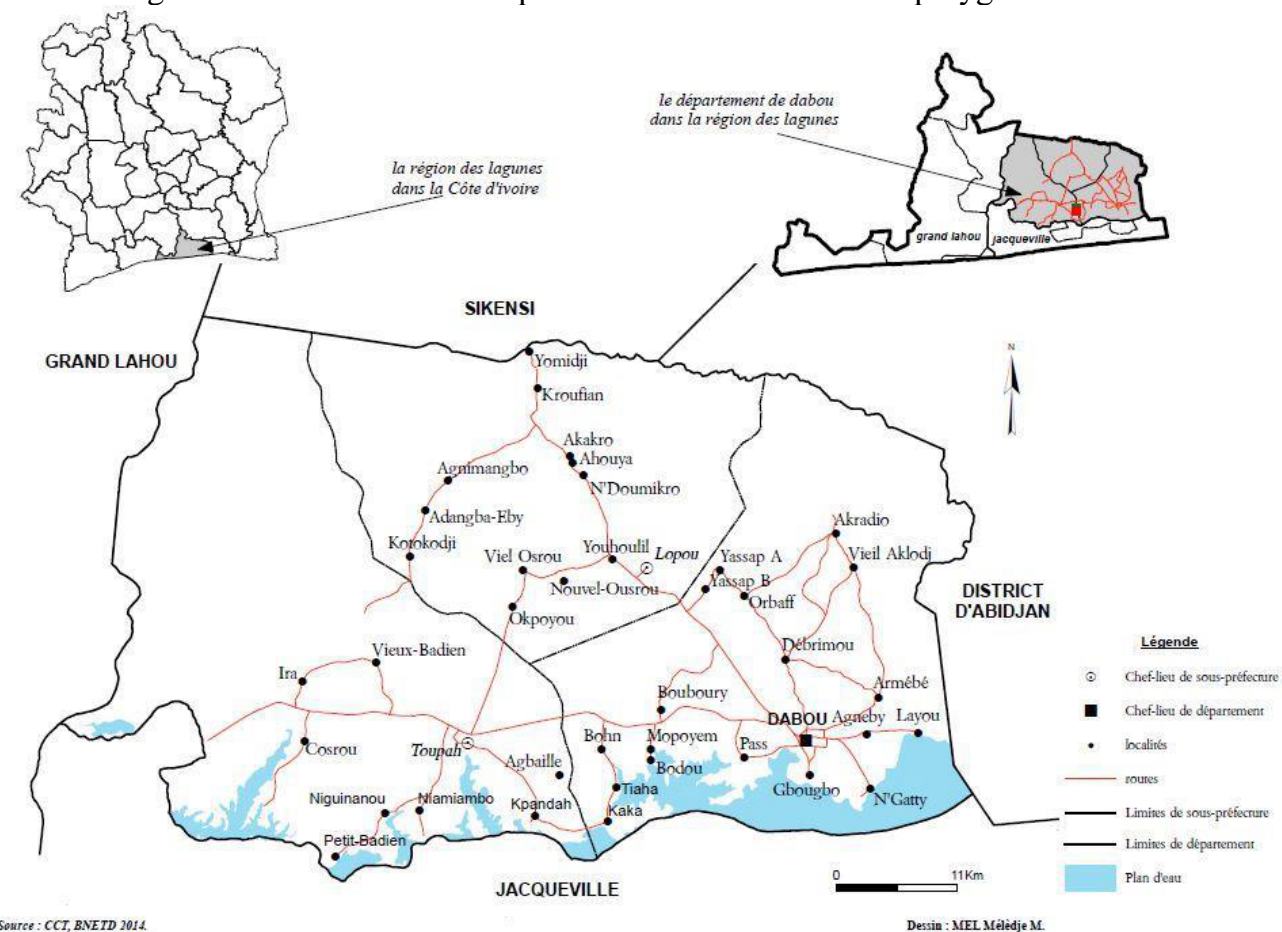

Seulement au sein de cet espace, ont été exclus de notre étude les villages relevant du groupe ethnique abidji (Yomidji, Kroufian, Agnimangbo, Adangba-Eby et Kotokodji).

L'échantillon constitué a donc été composé avec les villages de l'ethnie ôdjoukrou. À cet effet 12 localités ont été retenues sur les 35 existants. Les critères de sélection ont reposé sur la taille des localités et la position géographique (voir tableau 1).

Tableau 1 : Critères de sélection des localités

\begin{tabular}{|c|c|}
\hline \multirow{2}{*}{ Critères démographiques } & moins de 1000 habitants \\
\cline { 2 - 2 } & entre 1000 et 4000 habitants \\
\cline { 2 - 2 } & plus de 4000 habitants \\
\hline \multirow{2}{*}{ Position géographique } & avec façade lagunaire \\
\cline { 2 - 2 } & savane du Nord \\
\hline
\end{tabular}

Source : nos enquêtes de terrain

L'association desdits critères a abouti au choix de 11 localités sur les 35 qu'on retrouve dans le pays rural ôdjoukrou (figure 2). 
Figure 2 : Le pays ôdjoukrou et les localités objets d'enquête

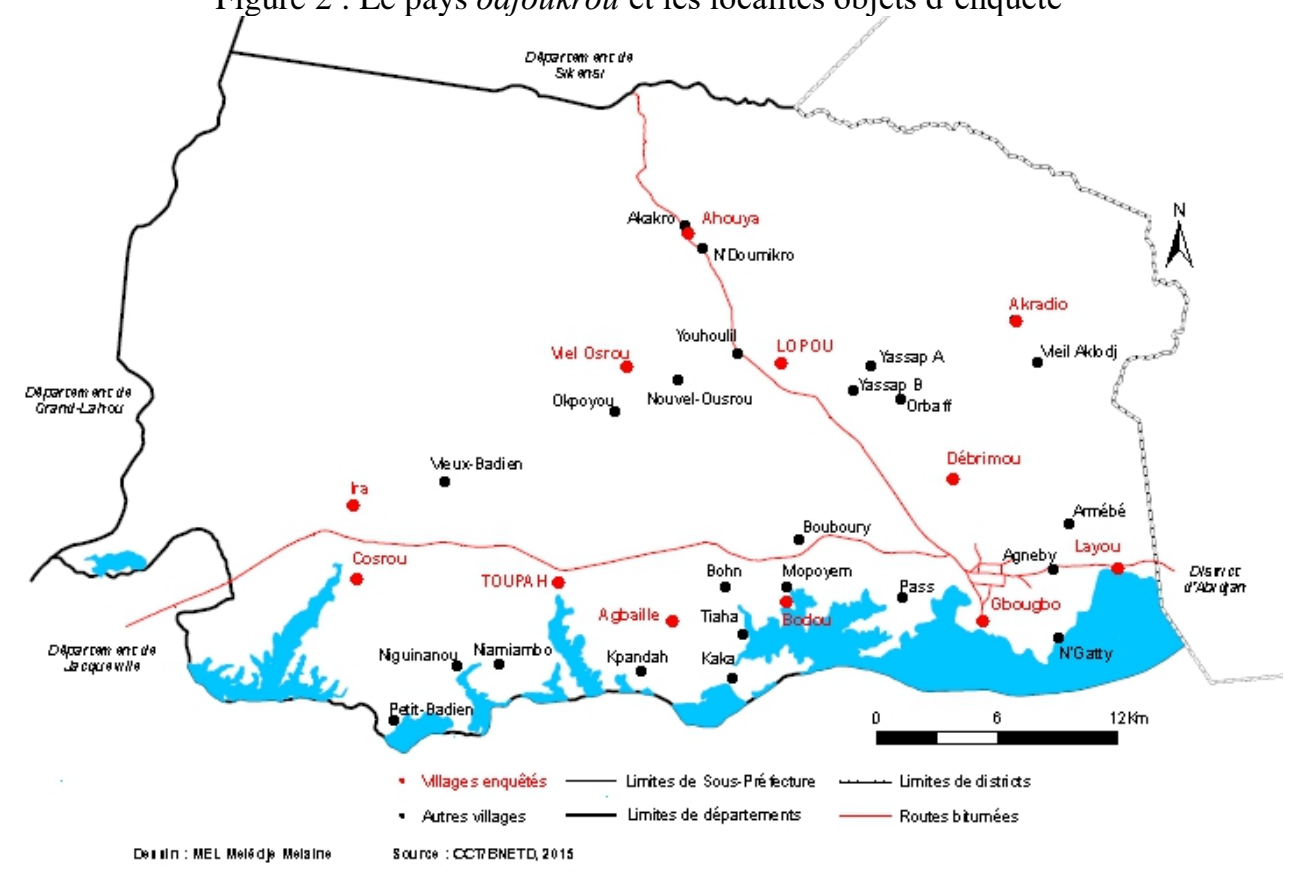

Dans chacune de ces localités un échantillon correspondant au 1/20 de la population de migrants a été réuni. Le tableau 2 nous présente la synthèse.

Tableau 2 : Migrants enquêtés par localités

\begin{tabular}{|c|c|c|c|c|}
\cline { 2 - 5 } \multicolumn{1}{c|}{} & Population totale & Population migrante & Echantillon au 1/20 & Echantillon harmonisé \\
\hline Ira & 4657 & 4603 & 230,15 & 230 \\
\hline Cosrou & 5485 & 3277 & 163,85 & 164 \\
\hline Toupah & 8028 & 6214 & 310,7 & 311 \\
\hline Agbaille & 1201 & 377 & 18,85 & 19 \\
\hline Lopou & 6702 & 1336 & 66,8 & 67 \\
\hline Ahouya & 702 & 304 & 15,2 & 22 \\
\hline Vieil Ousrou & 673 & 168 & 8,4 & 20 \\
\hline Akradio & 3906 & 994 & 49,7 & 50 \\
\hline Débrimou & 4250 & 435 & 21,75 & 22 \\
\hline Layou & 253 & 203 & 10,15 & 20 \\
\hline Gbougbo & 2179 & 1100 & 55 & 55 \\
\hline Bodou & 397 & 112 & 5,6 & 20 \\
\hline
\end{tabular}

Source : nos enquêtes, 2015

Les données collectées seront présentées sous forme de figures et de tableaux. 


\section{Résultats}

L'analyse des caractéristiques de la population migrante nous permet de présenter deux grandes dimensions du profilage que sont les aspects démographiques et économiques.

\section{- Caractéristiques démographiques des migrants}

Le critère démographique englobe l'étude selon le sexe, l'âge, le niveau d'instruction, le statut matrimonial, la taille des ménages, l'origine géographique et la religion des migrants.

\section{- L'analyse selon le sexe}

L'analyse des données montre que les hommes dominent l'immigration vers le pays rural ôdjoukrou. C'est ce constat que présente la figure 3 .

Figure 3 : Répartition des immigrants selon le sexe

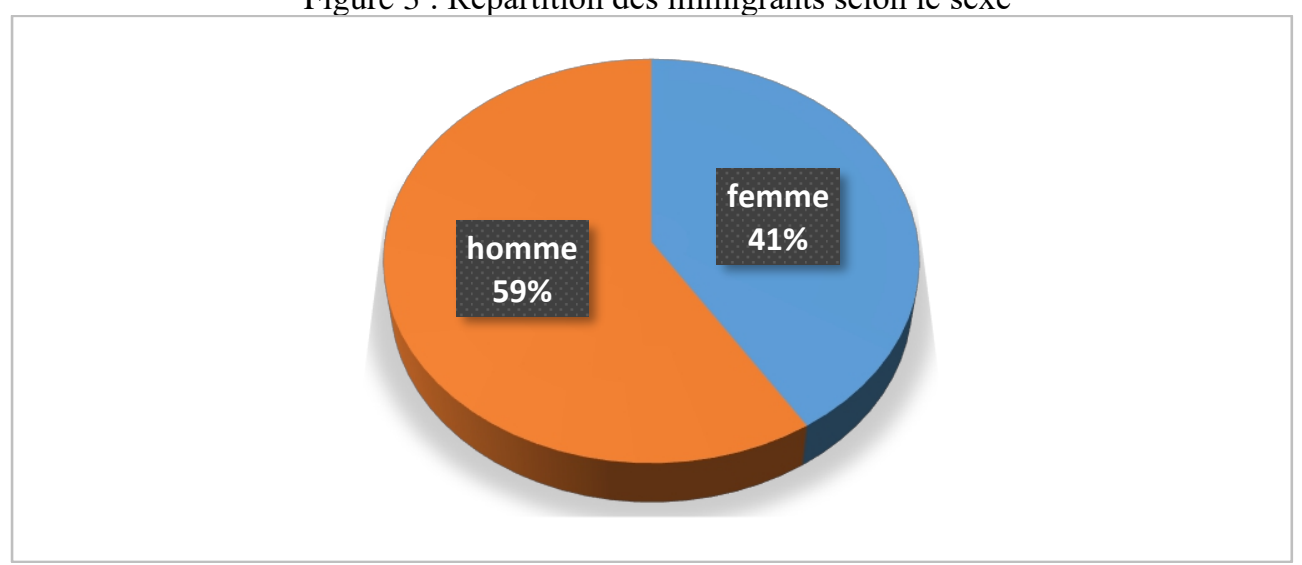

Source : notre enquête, 2015

Même si on note une présence relativement importante de la gente féminine avec un taux de $41 \%$, les hommes constituent la frange la plus importante avec 59\% de la population enquêtée. C'est un constat qui peut se justifier par le type d'activité dominante dans la zone, espace rural, l'agriculture a besoin de force physique en quantité et en qualité.

\section{- Que retenir de la question de l'âge ?}

Le constat dans ce cas de figure penche pour une prédominance des adultes dont l'âge est supérieur ou égale à 35 ans. Les informations contenues dans le tableau 3 corroborent cette version. 
Tableau 3 : Répartition des migrants en fonction de l'âge

\begin{tabular}{|c|c|c|c|}
\hline Tranche d'âge & homme & femme & Total \\
\hline $20-24$ & 31 & 48 & 79 \\
\hline $25-29$ & 20 & 14 & 34 \\
\hline $30-34$ & 29 & 23 & 52 \\
\hline $35-39$ & 48 & 38 & 86 \\
\hline $40-44$ & 251 & 155 & 406 \\
\hline $45-49$ & 158 & 106 & 264 \\
\hline $50-54$ & 10 & 19 & 29 \\
\hline $55-59$ & 25 & 07 & 32 \\
\hline $60-64$ & 11 & 00 & 11 \\
\hline 65 et plus & 07 & 00 & 07 \\
\hline Total & 590 & 410 & 1000 \\
\hline
\end{tabular}

Source : notre enquête, 2015

Les migrants dont l'âge est supérieur ou égale à 35 ans constituent la frange la plus importante de cette population. Ils représentent $78.7 \%$ des enquêtés.

\section{- Le niveau d'instruction}

Relativement à ce critère nous sommes parvenus à regrouper les migrants en trois catégories comme nous le présente le tableau 4.

Tableau 4 : Répartition des migrants en relation avec le niveau d'instruction

\begin{tabular}{|c|c|c|c|c|c|}
\hline Tranche d'âge & Sans niveau & Primaire & Secondaire & Supérieur & Total \\
\hline $20-24$ & 72 & 8 & 1 & 0 & 81 \\
\hline $25-29$ & 14 & 17 & 3 & 0 & 34 \\
\hline $30-34$ & 26 & 18 & 8 & 0 & 52 \\
\hline $35-39$ & 18 & 44 & 20 & 2 & 84 \\
\hline $40-44$ & 366 & 11 & 29 & 0 & 406 \\
\hline $45-49$ & 233 & 28 & 3 & 0 & 264 \\
\hline $50-54$ & 19 & 6 & 4 & 0 & 29 \\
\hline $55-59$ & 27 & 5 & 0 & 0 & 32 \\
\hline $60-64$ & 11 & 0 & 0 & 0 & 11 \\
\hline 65 et plus & 7 & 0 & 0 & 0 & 7 \\
\hline Total & 793 & 137 & 68 & 2 & 1000 \\
\hline
\end{tabular}

Source : notre enquête, 2015

Les résultats mettent en évidence un faible niveau d'instruction de la population migrante. Les illettrés constituent la frange la plus importante des migrants, soit $79,3 \%$.

\section{○ L'origine géographique}

Aujourd'hui les principales nationalités impliquées dans l'immigration vers lôdjoukrou sont restées quasiment identiques que celles héritées de la 
colonisation. Le tableau 5 nous apporte les détails sur l'origine géographique des migrants enquêtés.

Tableau 5 : Nationalité des migrants enquêtés

\begin{tabular}{|c|c|c|c|c|c|c|}
\hline Burkinabés & Maliens & Béninois & Togolais & Ghanéens & Ivoiriens & Total \\
\hline 358 & 75 & 187 & 138 & 40 & 202 & 1000 \\
\hline
\end{tabular}

Source : nos enquêtes, 2015

Depuis la période coloniale, la Côte d'Ivoire subie des migrations interrégionales et internationales marquée par le recrutement d'étrangers pour le développement de l'économie de plantation.

L'accession à l'indépendance en 1960 n'a pas marqué de rupture dans cette immigration car les autorités ivoiriennes avaient opté pour la politique d'ouverture sur l'extérieur avec les pays voisins. Ce qui a provoqué des vagues migratoires de ressortissants des pays limitrophes de la Communauté Économique des États de l'Afrique de l'Ouest (CEDEAO) en direction de la Côte d'Ivoire (WADJA 2012).

\section{- La taille des ménages}

Pour comprendre l'implication des migrants dans le processus de croissance démographique, la taille des ménages est un indicateur de poids. À cet effet, le tableau 6 révèle l'importante contribution des migrants.

Tableau 6 : Composition des ménages de migrants

\begin{tabular}{|c|c|c|c|c|c|c|c|c|c|c|}
\hline Taille moyenne du ménage & 1 & 2 & 3 & 4 & 5 & 6 & 7 & 8 & 9 & Total \\
\hline Effectif observé & 122 & 690 & 61 & 190 & 361 & 202 & 156 & 9 & 28 & $\mathbf{1 8 1 9}$ \\
\hline
\end{tabular}

Source : notre enquête, 2015

\section{- Le statut matrimonial}

Concernant le statut matrimonial des migrants, les résultats obtenus nous ont permis de réaliser la figure 4 . Il faut préciser que, dans le cadre de cet article, ont été considérés en union tous les hommes et toutes les femmes mariés, de façon formelle ou non, ainsi que ceux vivant en union consensuelle. 
Figure 4 : Statut matrimonial des migrants dans lôdjoukrou

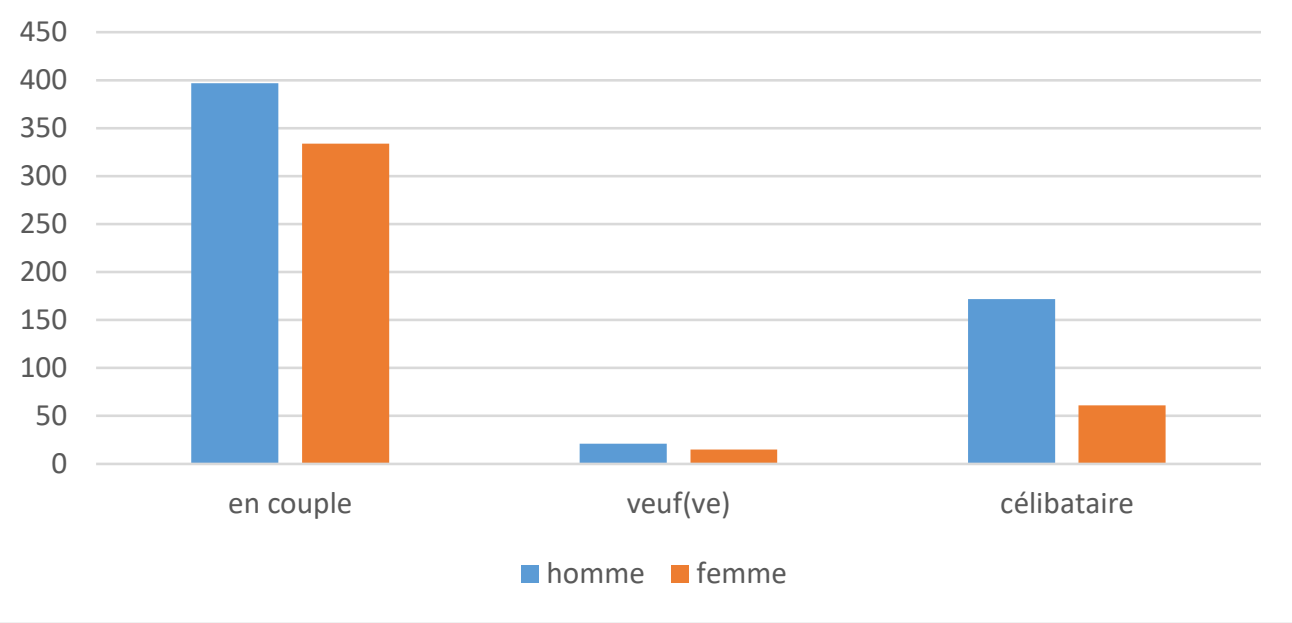

Source : nos enquête, 2015

Selon cette définition, on constate que la majorité des femmes $(81,46 \%)$ étaient en union au moment de l'enquête. À l'inverse, seulement $14,88 \%$ étaient célibataires. La proportion de femmes veuves représente 3,66 $\%$ des enquêtées. Chez les hommes, on observe une répartition de même type mais avec une proportion de célibataires $(29,15 \%)$ beaucoup plus élevée, cela peut s'expliquer par un âge à l'union beaucoup plus tardif chez les hommes que chez les femmes. Chez les hommes, 67,28 \% étaient en union au moment de l'enquête. Enfin, on remarque que la proportion d'hommes veufs $(3,55 \%)$ est quasiment identique que chez les femmes.

\section{- La religion}

Les données issues de la religion pratiquée par les migrants ont fait l'objet de représentation sur la figure 5 .

Figure 5 : Répartition des migrants par rapport à la religion

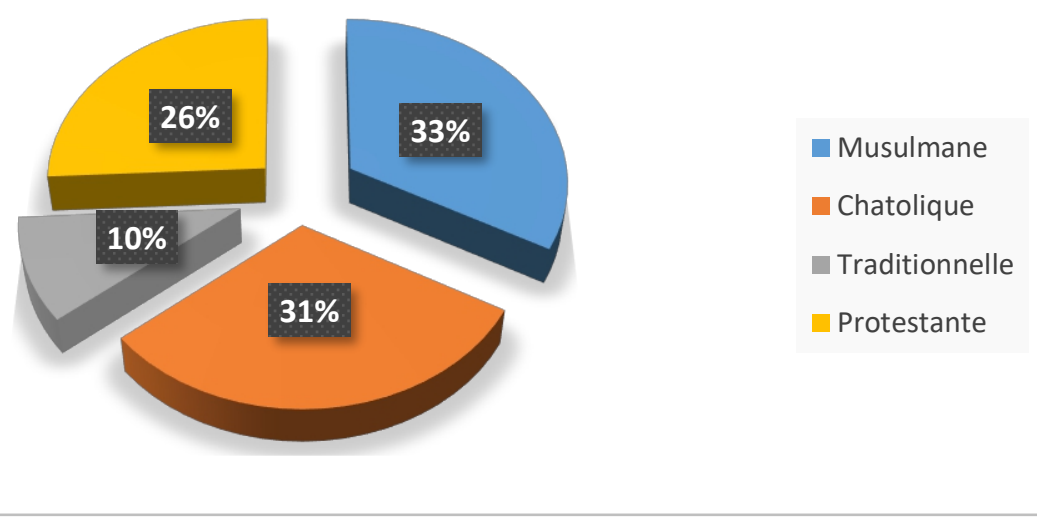

Source : nos enquêtes, 2015 
Selon les données recueillies sur la religion pratiquée par les migrants, on constate qu'un migrant sur trois (33\%) est de confession musulmane. Les Catholiques représentent $31,1 \%$ de cette population. Un peu plus du quart des migrants, soit $25,8 \%$ du total enquêté est Protestant et $10,1 \%$ des migrants ont déclaré pratiquer la religion animiste.

\section{- Caractéristiques économiques des migrants}

Les caractéristiques économiques évoquent les questions liées à l'emploi, au mode d'accession à la terre et au revenu.

\section{- Le type d'emploi des migrants}

L'enquête a permis de collecter des informations relatives à l'emploi des migrants hommes et femmes. Selon la définition que nous avons adoptée, est considérée comme ayant un emploi, toute personne ayant déclaré une activité régulière ou non dans le secteur formel ou informel, avec une contrepartie financière. La figure 6 donne la répartition des migrants selon le domaine dans lequel il exerce.

Figure 6 : Effectifs des migrants par secteurs d'activités

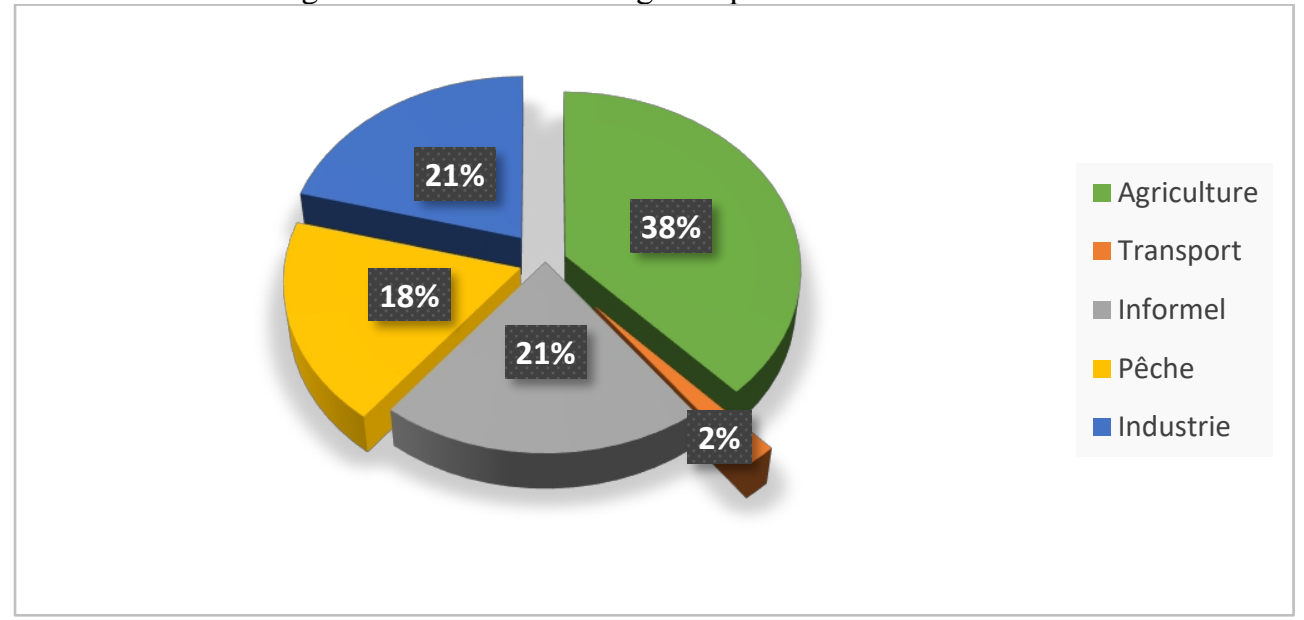

Source : nos enquêtes, 2015

Ramenées au rapport hommes-femmes (tableau 7), puis mis en relation avec l'origine géographique des migrants (figure 7), les données relatives à l'emploi se révèlent plus détaillées.

Tableau 7 : Répartition selon le genre et le type d'activité des migrants

\begin{tabular}{|c|c|c|c|c|c|c|}
\cline { 2 - 7 } \multicolumn{1}{c|}{} & Agriculture & Transport & Informel & Pêche & Industrie & Total \\
\hline hommes & 244 & 18 & 7 & 186 & 135 & 590 \\
\hline femmes & 136 & 0 & 201 & 0 & 73 & 410 \\
\hline Total & 380 & 18 & 208 & 186 & 208 & 1000 \\
\hline
\end{tabular}

Source : nos enquêtes, 2015 
L'analyse des résultats liés à l'activité économique met en évidence un certain nombre de disparités et une spécialisation de la migration relativement au type d'activité exercée.

Comme on pouvait s'y attendre, l'agriculture avec $38 \%$ est l'activité majoritairement partagée par les migrants. Le transport n'occupe que $2 \%$ de cette population. Entre ces deux extrêmes, l'informel et l'industrie arrivent à égalité avec $21 \%$ chacun puis $18 \%$ pour la pêche.

Excepté le transport et la pêche où leurs absence est fortement remarquable, les femmes sont présentes dans l'agriculture $(35,79 \%)$, elles dominent l'informel $(96,63 \%)$ et font une percée remarquée dans l'industrie $(35,09 \%)$. Les hommes sont eux disséminés dans l'ensemble du domaine économique.

Figure 7 : Répartition des migrants selon la nationalité et le types d'activité

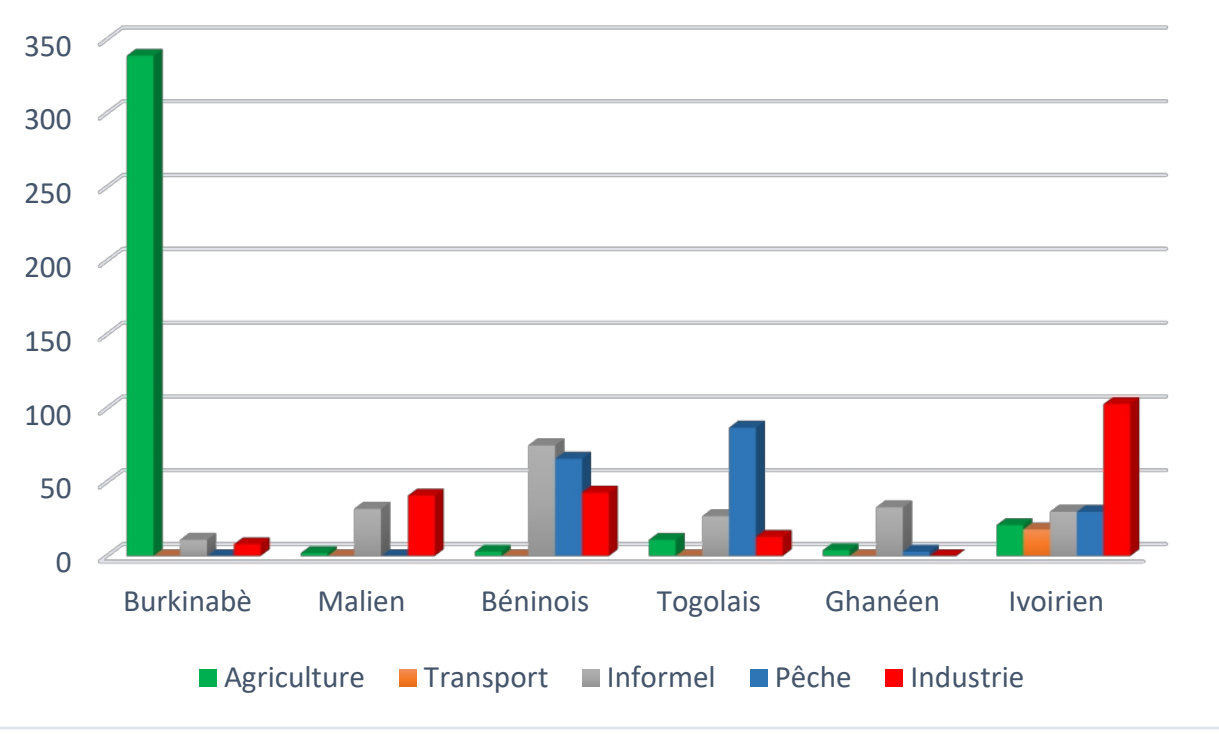

Source : nos enquêtes, 2015

Il est possible de mettre en relation l'origine géographique des migrants et les principaux secteurs d'activités qui les accueillent. Ainsi, si les allochtones sont représentés dans l'ensemble de la sphère économique, on note qu'ils dominent dans l'industrie où ils représentent presque un travailleur sur deux $(49,51 \%)$. La pêcherie est aujourd'hui l'exercice privilégié par les Togolais (46,77\%) et Béninois (35,48\%), ensemble ils représentent (82,25\%) des migrants et ont pour seuls concurrents les autochtones ôdjoukrou. Mais c'est au sein de l'agriculture avec 94,21\% des Burkinabés que cette perception de notre analyse prend tout son sens. D'où la question lancinante du mode d'accession à la terre. 


\section{- Le mode d'accession à la terre}

La pratique de l'agriculture nécessite une possession de terre à usage agricole. Les migrants en théorie ne disposent pas de terre, mais cette situation peut être contournée par ces derniers. La terre peut être soit à usage propre, dans ce cas le migrant est "propriétaire" ( $37 \%$ des cas enregistrés) ou le migrant peut travailler pour le compte d'une tierce personne (63\% des cas comptabilisés). Dans le cadre d'une propriété du migrant les options d'acquisition de la terre sont illustrées par la figure 8 .

Figure 8 : Importance des modes d'accession à la terre pour les migrants

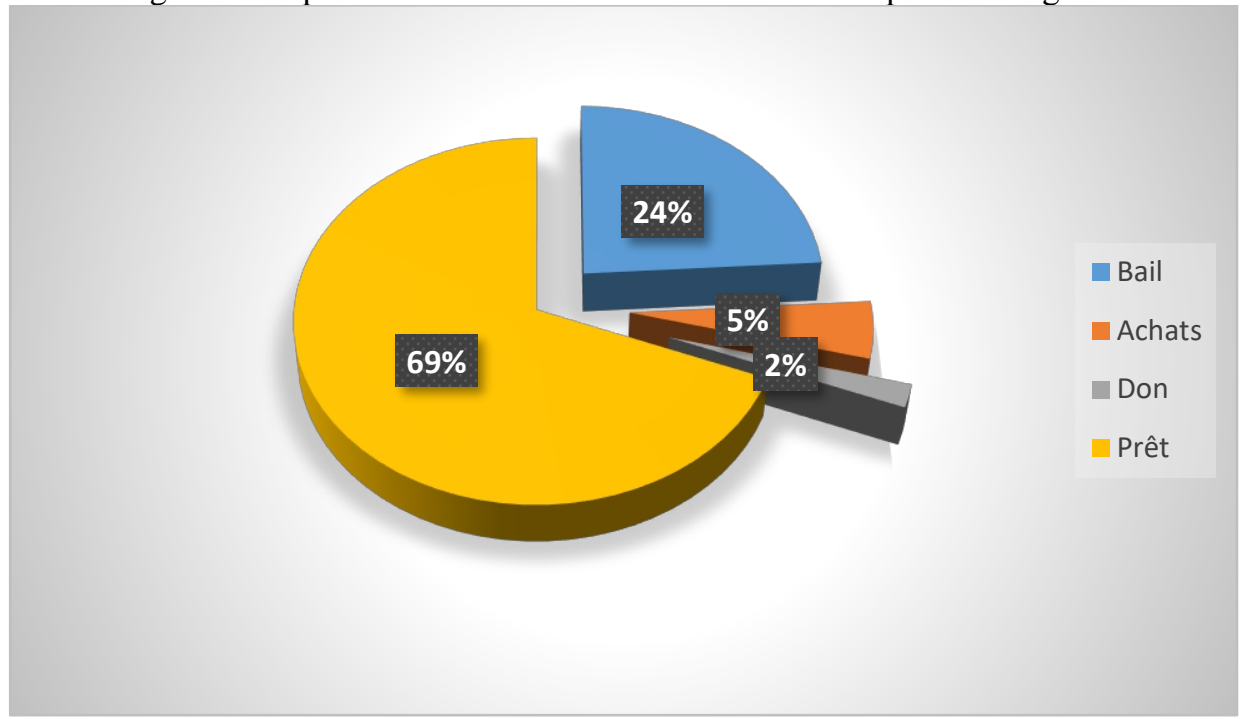

Source : nos enquêtes, 2015

\section{- L'analyse du revenu}

Si l'agriculture est l'activité dominante, divers acteurs y interviennent. À partir de l'an 2000, le prix d'achat du latex connaît une importante hausse (jusqu'à $1200 \mathrm{FCFA} / \mathrm{kg}$ ). Les mieux rémunérés travaillent alors dans la collecte du latex. Aujourd'hui avec la chute du prix (en 2017 le prix moyen était de $250 \mathrm{FCFA} / \mathrm{kg}$ ), ces derniers perçoivent un salaire moyen de 80000 FCFA. Un ouvrier agricole perçoit entre 2000 et 2500 FCFA/ jour, soit un maximum de 75000 FCFA mensuel. Une majorité de migrant, 67\% des cas étudiés s'estiment satisfait, seul 4\% des migrants sont insatisfaits de leur situation économique et envisagent une nouvelle émigration. La frange intermédiaire, soit $29 \%$ des migrants, reste optimiste en un lendemain meilleur au sein de l'espace d'accueil. Néanmoins, ce n'est pas moins de $82 \%$ des migrants qui transfèrent mensuellement des fonds à destination de la famille restée dans l'espace de départ. 


\section{Discussion}

L'actualité médiatique dans le monde porte sur les migrations en ce début de $\mathrm{XXI}^{\mathrm{ème}}$ siècle. Au regard des commentaires qui en ressortent, nous pouvons souligner que la population africaine est un des leviers de la question des migrations dans le monde. À ce propos (GREGORY, 1988) fait remarquer que l'histoire des migrations en Afrique au Sud du Sahara peut être découpée en trois grandes étapes. Du XVII ${ }^{\text {ème }}$ au XIX ${ }^{\text {ème }}$ siècle la traite des esclaves domine; entre 1880 et 1945, la pénétration coloniale accroît les besoins de main-d'œuvre (travail forcé) et entraîne l'implantation de quelques places fortes, les villes-comptoirs. Pendant l'époque coloniale également, certaines migrations intra-africaines ont été favorisées afin d'accélérer le développement économique de certaines régions stratégiques, c'est 1'exemple du polygone ivoirien en général et de son littoral en particulier qui continu d'accueillir une importante population issue de l'immigration. Pour parler du littoral ivoirien, SALE (2004) souligne le fait qu'on y enregistre une forte concentration humaine et celle d'intenses activités économiques. En effet, les espaces péri lagunaires de la Côte d'Ivoire sont des lieux éminemment stratégiques qui attirent les migrants.

Ils constituent de fait l'objet de grandes convoitises. Lôdjoukrou, présenté dans cet article en tant qu'espace d'accueil est un espace qui s'inscrit dans le littoral ivoirien.

De par les mutations nombreuses et variées qu'il subit, l'espace rural ôdjoukrou, connait un fonctionnement qui se complexifie. Les migrants qu'il accueille sont de plus en plus mis en avant quand on décrypte les causes qui concourent à ces changements. C'est ce que PIERMAY (2003) identifie comme élément «révélateur» il souligne que c'est un «ensemble de processus clairement identifiables, porteurs d'enjeux forts et susceptibles d'éclairer les pratiques, les stratégies et les intérêts des différents acteurs qui interviennent dans le champ étudié».

L'immigration ici présente un caractère profondément ouest africain. Cette coloration sous régionale de l'immigration trouve son explication, selon DUPIRE et BOUTILLER (1958), dans le processus colonial qui a participé de la fixation d'une importante population servile relativement aux besoins en mains d'œuvre exerçant dans les plantations industrielles. MEMEL-FOTE (1980) note que ce désidérata des colons est à l'origine d'une non moins importante diaspora. Ces diasporas tissent des réseaux communautaires actifs, fortement intégrés, et économiquement organisés. Elles stimulent les mouvements migratoires au sein des communautés. Sous une autre approche, il y a aussi l'institution qu'est la Communauté Économique Des États d'Afrique de l'Ouest, qui en théorie supprime les frontières pour les populations des États membres, enfin il y a la relative accessibilité des espaces, 
aujourd'hui interconnectés par les systèmes de transports. D'où la priorité des pays au voisinage de la Côte d'Ivoire.

L'implication des migrants dans la sphère économique est l'un des aspects de leur intégration dans une société devenue hétérogène. Le facteur nouveau de leur implication dans l'économie agricole tient à des formes évoluées de la propriété de la terre. Pour SEGBENOU et alii, (2013) cela tient de la logique actuelle, le littoral ôdjoukrou fait partie des espaces où l'acquisition de terres par les migrants est le plus important. À en croire COULIBALY (2008) la logique doit être perçue à une autre échelle, celle qui stipule que dans la plupart des cas en Côte d'Ivoire ce sont les étrangers qui tiennent l'économie dans l'espace d'accueil.

\section{Conclusion}

La population migrante au sein du pays rural ôdjoukrou présente un caractère varié. En nous fiant à l'analyse de nos données de terrain et de ceux des résultats du RGPH 2014, nous pouvons conclure à un processus de perpétuelle accumulation de cet effectif. Aujourd'hui, les migrants représentent plus de $40 \%$ du total de la population rurale dans lodjoukrou. Ce chiffre porte à réflexion car il est largement supérieur à la moyenne nationale qui est de $26 \%$. Cela prouve que lodjoukrou est un espace sous emprise migratoire. La majorité de ces migrants proviennent de la sous-région ouest africaine, avec une prédominance de la diaspora Burkinabè, avec un taux de $36 \%$. Le caractère sous régional de cette migration est la conséquence des politiques coloniales et postes coloniales de transhumance ouvrières. À cela il faut ajouter le fait que lôdjoukrou soit devenu attractif à cause de sa position géographique préférentielle proche d'Abidjan la capitale économique ivoirienne, mais surtout de son importante activité agricole qui peut fournir du travail à tous.

\section{References:}

1. COULIBALY Kélémory, 2008, Anyama, une ville "Malinké" en pays Akyé, Thèse unique de géographie, IGT, Université de CocodyAbidjan, 419p.

2. DUPIRE Marguerite et BOUTILLIER Jean-Louis. 1958, Le pays Adioukrou et sa palmeraie (basse-côte-d' ivoire), L'HOMME D'OUTRE-MER, n4, $101 \mathrm{p}$.

3. INS, 2014, État et structure de la population, RGPH, volume 4, tome 1, Abidjan, $118 \mathrm{p}$.

4. KABLAN N'GUESSAN H. Y., 2005, La surveillance des côtes ivoiriennes, In : GEOTROPE, n ${ }^{\circ}$, EDUCI, Abidjan, pp 3-15. 
5. MEMEL-FOTÊ Harris, 1980, Le système politique de lôdjoukrou, éditions Présence Africaine et nouvelles éditions africaines, Abidjan, $479 \mathrm{p}$.

6. OIM. 2009, Migration en Côte d'Ivoire: Profil national 2009, L'avenir des migrations : Renforcer les capacités face aux changements. $121 \mathrm{p}$.

7. PIERMAY J.L, 2003, L'invention de la ville en Afrique subsaharienne, In : Historiens

8. et Géographes, $\mathrm{n}^{\circ} 379$, pp. 24-41.

9. SALE Ane, 2004, Etat actuel du milieu lagunaire ivoirien : une approche par télédétection et les SIG, Communication de l'atelier de Lagos, Nigeria, $17 \mathrm{p}$.

10. SEGBENOU René et alii., 2013, Étude participative sur les acquisitions massives de terres agricoles en Afrique de l'ouest et leur impact sur l'agriculture familiale et la sécurité alimentaire des populations locales. Zone d'étude : Côte d'Ivoire, rapport d'enquête. $53 \mathrm{p}$.

11. WADJA J., 2012, Dynamique du peuplement et transformation de l'espace dans la région du Bas Sassandra (Sud-Ouest de la Côte d'Ivoire), Thèse unique, IGT, $315 \mathrm{p}$. 